\title{
Analysis of Access and Connectivity Probabilities in Vehicular Relay Networks
}

\author{
Seh Chun Ng, Student Member, IEEE, Wuxiong Zhang, Yu Zhang, Yang Yang, Senior Member, IEEE, \\ and Guoqiang Mao, Senior Member, IEEE
}

\begin{abstract}
IEEE 802.11p and 1609 standards are currently under development to support Vehicle-to-Vehicle and Vehicleto-Infrastructure communications in vehicular networks. For infrastructure-based vehicular relay networks, access probability is an important measure which indicates how well an arbitrary vehicle can access the infrastructure, i.e. a base station (BS). On the other hand, connectivity probability, i.e. the probability that all the vehicles are connected to the infrastructure, indicates the service coverage performance of a vehicular relay network. In this paper, we develop an analytical model with a generic radio channel model to fully characterize the access probability and connectivity probability performance in a vehicular relay network considering both one-hop (direct access) and two-hop (via a relay) communications between a vehicle and the infrastructure. Specifically, we derive close-form equations for calculating these two probabilities. Our analytical results, validated by simulations, reveal the tradeoffs between key system parameters, such as inter-BS distance, vehicle density, transmission ranges of a $\mathrm{BS}$ and a vehicle, and their collective impact on access probability and connectivity probability under different communication channel models. These results and new knowledge about vehicular relay networks will enable network designers and operators to effectively improve network planning, deployment and resource management.
\end{abstract}

Index Terms - Vehicular Ad Hoc Network (VANET), Wireless Access in Vehicular Environments (WAVE), IEEE 802.11p, IEEE 1609, access probability, connectivity, relay.

Manuscript received 5 January 2010; revised 7 May 2010 and 12 July 2010. This work is partially supported by the Australian Research Council (ARC) under the Discovery project DP0877562, the National Natural Science Foundation of China (NSFC) under the grant 60902041, the Ministry of Science and Technology (MOST) of China under the grant 2009DFB13080, and the RCUK project "UK-China Science Bridges: R\&D on (B)4G Wireless Mobile Communications (EP/G042713/1)". This work is partially supported by National ICT Australia (NICTA), which is funded by the Australian Government Department of Communications, Information Technology and the Arts and the Australian Research Council through the Backing Australia Ability initiative and the ICT Centre of Excellence Program, and by the Air Force Research Laboratory, under agreement number FA2386-10-1-4102. The U.S. Government is authorized to reproduce and distribute reprints for Governmental purposes notwithstanding any copyright notation thereon. The views and conclusions contained herein are those of the authors and should not be interpreted as necessarily representing the official policies or endorsements, either expressed or implied, of the Air Force Research Laboratory or the U.S. Government.

S. C. Ng and G. Mao are with School of Electrical and Information Engineering, University of Sydney, Australia and National ICT Australia (NICTA), Sydney (e-mail: \{sehchun,guoqiang\}@ee.usyd.edu.au).

W. Zhang is with Shanghai Research Center for Wireless Communications (WiCO), Graduate School of the Chinese Academy of Sciences, China (email: wuxiong.zhang@shrcwc.org).

Y. Zhang is with the Dept. of Computing, Imperial College London, UK (e-mail: yuzhang@doc.ic.ac.uk).

Y. Yang is with Shanghai Research Center for Wireless Communications (WiCO), Shanghai Institute of Microsystem and Information Technology (SIMIT), Chinese Academy of Sciences, China (e-mail: yang.yang@shrcwc.org) corresponding author.

Digital Object Identifier 10.1109/JSAC.2011.110114.

\section{INTRODUCTION}

$\mathbf{V}$ EHICULAR ad-hoc network (VANET) is a type of promising application-oriented network deployed along a highway for safety and emergency information delivery (for drivers), entertainment content distribution (for passengers), and data collection and communication (for road and traffic managers). VANET is a hybrid wireless network that supports both infrastructure-based and ad hoc communications. Specifically, vehicles on the road can communicate with each other through a multi-hop ad hoc connection. They can also access the Internet and other broadband services through the roadside infrastructure, i.e. base stations (BSs) or access points (APs) along the road. When a vehicle moves out of the radio coverage area of a BS, e.g. it is located in the coverage gap between two adjacent BSs, it will identify and use its neighboring vehicles (if any) as relays to access the roadside infrastructure. These types of Vehicle to Vehicle (V2V) and Vehicle to Infrastructure (V2I) communications have recently received significant interests from both academia and industry [1], [2], [3]. V2V communication has so far been envisioned for supporting safety and traffic management applications. With better sensing and data communication techniques, drivers can share the information such as slippery road, poor visibility, sudden stop and road congestion with each other. Hence warnings are provided to prevent accidents and improve road safety.

As shown in Fig. 1, IEEE 802.11p standard cooperates with the IEEE 1609 standard family, which is developed to support Wireless Access in Vehicular Environment (WAVE) and to deliver safety and infotainment applications to vehicles on the road [4], [5]. Specifically, IEEE 802.11p is a draft amendment (with WAVE capability) to the IEEE 802.11 standards, which is expected to be finalized and approved in 2010. The goal of $802.11 \mathrm{p}$ standard is to provide $\mathrm{V} 2 \mathrm{~V}$ and V2I communications, up to a range of $1 \mathrm{~km}$, at an average data rate of $6 \mathrm{Mbps}$ over the dedicated $5.9 \mathrm{GHz}(5.85-5.925 \mathrm{GHz})$ licensed frequency band. IEEE 802.11p uses an amended 802.11a physical-layer specification with Orthogonal Frequency-Division Multiplexing (OFDM) technique. On Medium Access Control (MAC) layer, it adopts the Enhanced Distributed Channel Access (EDCA) protocol from the 802.11e standard to support Quality of Service $(\mathrm{QoS})$. The following standards are included in the IEEE 1609 standard family: IEEE P1609.0, IEEE P1609.1, IEEE P1609.2, IEEE P1609.3, IEEE P1609.4. Some new standards have recently been added to IEEE 1609 family, such as 1609.5 (Communications Management), 1609.6 (Facilities) and 1609.11 (Electronic Payment Service). Their functions and relationships with other 1609 standards are shown in Fig. 1. 


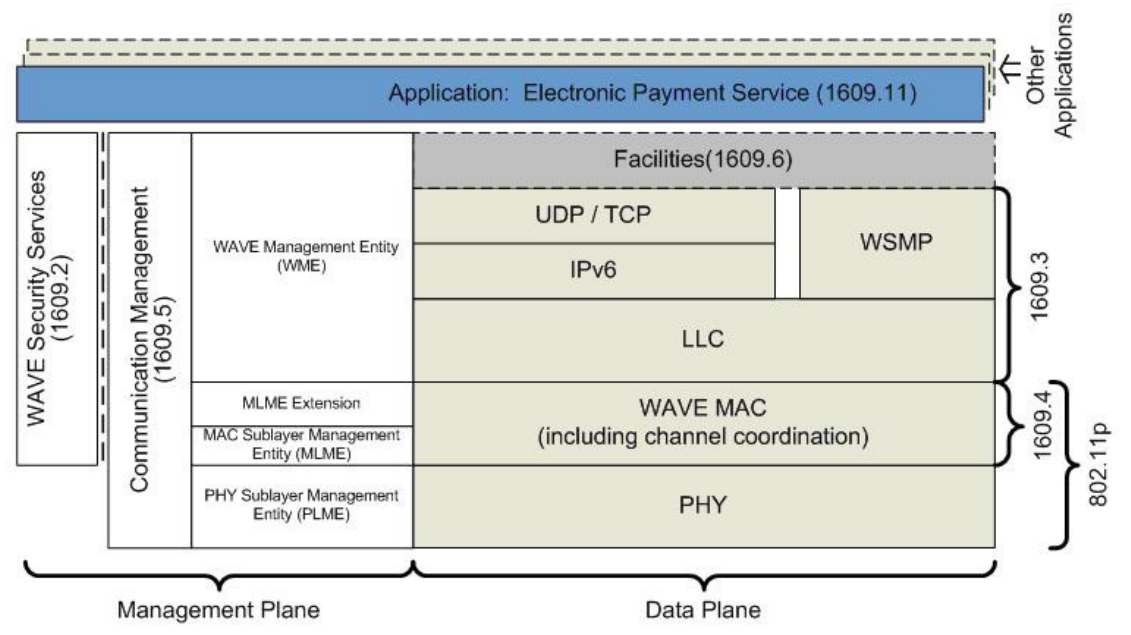

Fig. 1. Architecture of IEEE 1609 standards family

IEEE $802.11 \mathrm{p}$ and 1609 standards are still in the draft stage. The harsh vehicular communication environment, caused by variable vehicle speed, high mobility, and dynamic network topology, brings many technical challenges in developing and deploying WAVE applications and services. From a user/vehicle's perspective, the first, and probably the most important, service requirement is to be able to access the roadside infrastructure, directly or indirectly via a relay vehicle. From the perspective of a network operator or service provider, it is important to guarantee satisfactory and profitable service coverage while minimizing the deployment and maintenance costs of the roadside infrastructure.

To improve user satisfaction and service coverage of future IEEE 1609 based WAVE systems and applications, this research develops an analytical model to fully characterize the access probability (for user satisfaction analysis) and the connectivity probability (for service coverage analysis) for infrastructure-based vehicular relay networks, wherein both one-hop (direct access) and two-hop (via a relay) communications between a vehicle and the infrastructure (i.e. a BS) are supported. In this paper, a generic connection model is used to investigate the impact of different system parameters, i.e., inter-BS distance (or BS density), vehicle density, radio coverage ranges of BSs and vehicles, on key performance metrics, i.e. user access probability and service connectivity probability. The analysis is then applied to two widely used communication channel models as specific examples of the generic connection model. This research enables us to improve access probability and connectivity probability in vehicular relay networks, and therefore support reliable V2I and V2V data transmissions in different commercial applications and services, such as emergency messaging service, mobile Internet access and on-road entertainments.

The rest of this paper is organized as follows. In Section II we introduce related work. In Section III we define the system model. In Section IV we present the analysis of the access and connectivity probabilities under a generic radio channel model. In Section $\mathrm{V}$ we focus on two widely used radio channel models, i.e. the unit disk communication model and the lognormal shadowing model, and their analysis as special cases of the generic channel model. In Section VI we discuss the analytical and simulation results, followed by conclusions in Section VII.

\section{RELATED WORK}

Recently, significant research on VANET, WAVE, IEEE 802.11p and 1609 standards has been undertaken to measure, estimate and characterize wireless vehicular channels [6], [7], [8], to model and analyze system performance [9], [10], [11], [12], [13], [14], to design and evaluate MAC protocols [15], [16], [17], [18], [19], and to develop VANET simulator [20] and testbed [21]. Specifically, in [9], it is found a communication distance of $1000 \mathrm{~m}$, which is specified in the IEEE802.11p Project Authorization Request (PAR), cannot be achieved by an Equivalent Isotropically Radiated Power (EIRP) of $2 \mathrm{~W}$ in a vehicle-to-vehicle (V2V) highway scenario. The impacts of vehicle density (or V2V distance) and Line-Of-Sight (LOS) communication link on different system performance metrics, such as throughput, average delay, packet loss, and collision probability, are investigated in [10], [11], [12].

In [15], it is pointed out that the performance of IEEE $802.11 \mathrm{p}$ standard is not satisfactory in the infrastructure data collection mode with a static backoff scheme. The capability of 802.11p MAC protocol is further evaluated and enhanced to support both safety applications (i.e. emergency message dissemination with strict time constraints) and non-safety applications [16], [17], [22], [18], [19]. In [23], Salhi et al. presented a novel data gathering and dissemination architecture based on hierarchical and geographical mechanisms for vehicular sensor networks. In [24] and [25] practical traffic prioritization and power control schemes are proposed and evaluated respectively, to support real-time delivery of safetycritical emergency information. In [26], Shrestha et al. develop a new scheme using the BitTorrent tool and bargaining game to efficiently distribute a large amount of data over $\mathrm{V} 2 \mathrm{~V}$ and V2I communication links.

Access and connectivity probabilities have been studied in the literature for one-dimensional (1-D) [27], [28] and two-dimensional (2-D) [29], [30], [31] multi-hop wireless networks. In [27], Wu focuses on V2V communications and derives a close-form expression of connectivity probability in a linear VANET with high-speed vehicles and time-varying 
vehicle populations, i.e. dynamic network topology and vehicle density. The impacts of some key network parameters, such as vehicle arrival rate, random vehicle speed and transmission range, are considered in his work. Based on a Poisson assumption of node distribution, Dousse, Thiran and Hasler study a 1-D network with equally-spaced BSs and Poissonly distributed vehicles in [28]. Considering a unit disk communication model, they derive the connectivity probability (defined as access probability in this paper) that an arbitrary vehicle can reach at least one BS over multiple hops.

For a 2-D multi-hop cellular network, where nodes are uniformly distributed in a circular area of unit radius, Ojha et al. obtain the minimum transmission range required for these nodes to be able to access a BS located at the center of this circular area over multiple hops under the unit disk communication model as the number of nodes goes to infinity [29]. When both BSs and nodes are Poissonly distributed in a 2-D area and a log-normal shadowing communication model is considered, a lower bound on the probability that a node cannot access any BS within a designated number of hops has been derived in [30]. The result relies on the independence assumption that the event that a node cannot access to a BS in a specific number of hops, say $t$, and the event that another node cannot access to a BS in $t$ hops are independent, which is not always valid for some cases (to be shown in this paper). In [31], the probability that a wireless ad-hoc network with randomly and uniformly distributed nodes form a connected network is studied. It is shown that the probability of having a connected network and the probability of having no isolated node asymptotically converges to the same value as the number of nodes in the network goes to infinity.

Different from previous work carried out mainly under the unit disk communication model and considering no restriction on the maximum hop count for packet transmission, this research focuses on vehicular relay networks with a maximum hop count of two hops to ensure high communication quality and reliability between a node and a BS, i.e. two-hop V2I communications, which is more practical for real-world VANET application scenarios. In addition, our analytical approach uses a generic communication channel model and derives the exact close-form equations of access and connectivity probabilities, not the asymptotic results that are valid only when the number of nodes (or node density) in a network is very large. The results obtained under the generic channel model are then applied to two widely used models, i.e. the unit disk model and the log-normal model, as special cases. Finally, we investigate the impacts of some key system parameters, such as interBS distance (or BS density), vehicle density, transmission ranges of a BS and a vehicle, on user access probability and service connectivity probability performance under different communication channel models.

\section{System Model}

We consider an infrastructure-based vehicular relay network, as shown in Fig. 2, wherein a number of BSs are uniformly deployed along a long road, while other vehicles are distributed on the road randomly according to a Poisson distribution. We analyze the access probability, i.e. the probability that an arbitrary vehicle can access its nearby BSs within

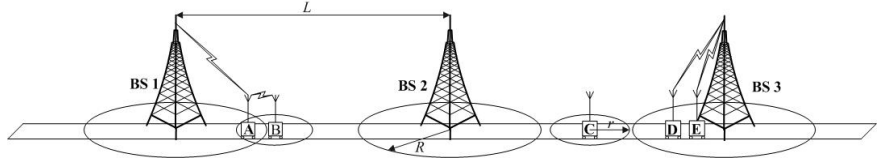

Fig. 2. An Infrastructure-based Vehicular Relay Network.

two hops, and the connectivity probability, i.e. the probability that all vehicles can access at least one BS within two hops, of the network by investigating a subnetwork bounded by two adjacent base stations. Let $L$ be the Euclidean distance (in meters) between two adjacent BSs and $\rho$ be the vehicle density measured in vehicles per meter (vpm). Since the vehicles are assumed to be Poissonly distributed with density $\rho$, discussion on the distribution of the number of vehicles on the road is only meaningful if we restrict to the (random) number of vehicles in a specific section of the road, and we call any section of the road a road segment. For a road segment with length $x$, the number of vehicles on that road segment is then a Poisson random variable with mean $\rho x$. So the probability that there are $k$ vehicles on a road segment of $x$ meters is given as

$$
f(k, x)=\frac{(\rho x)^{k} e^{-\rho x}}{k !}, k \geq 0 .
$$

Since we investigate a subnetwork bounded by two adjacent $\mathrm{BSs}$, the probability that there are $k$ vehicles on the road segment bounded by two adjacent BSs is then $f(k, L)$.

Assuming a generic channel model $\mathcal{C}$, let $g_{v}^{\mathcal{C}}(x)$ be the probability that two vehicles separated by an Euclidean distance $x$ are directly connected. Similarly, denote by $g_{b}^{\mathcal{C}}(x)$ the probability that a vehicle and a BS separated by an Euclidean distance $x$ are directly connected. We assume that the event that two vehicles (or a vehicle and a BS) are directly connected is independent of the event that another two vehicles (or a vehicle and a BS) are directly connected. That is, the event that two vehicles (or in the similar case, between a vehicle and a BS) are directly connected is only determined by the locations of the two vehicles and is not affected by the presence or absence of connections between other pairs of vehicles ${ }^{1}$. We also assume that $g_{b}^{\mathcal{C}}(x) \geq g_{v}^{\mathcal{C}}(x)$. This assumption is justified because it is often the case that a BS can not only transmit at a larger transmission power than a vehicle, it can also be equipped with more sophisticated antennas, which make it more sensitive to the transmitted signal from a vehicle.

\section{Analysis of ACCESS And ConNectivity PROBABILITIES}

Assume that the subnetwork being considered is placed at $[0, L]$. The two BSs at both ends of the subnetwork are labeled

\footnotetext{
${ }^{1}$ Although field measurements in real applications seem to indicate that the connectivity between different pairs of geographically / frequency proximate wireless nodes are correlated [32], [33], [34], the independence assumption is generally considered appropriate for far-field transmission and has been widely used in the literature under many channel models including lognormal shadowing model [35], [36], [30], [37]. Note that the unit disk model is a special channel model which fulfills the independence assumption by nature [38, pg. 12]. This is because for the unit disk model, two vehicles are directly connected if and only if their Euclidean distance is smaller than the transmission range, and is not affected by the presence or absence of other connections (vehicles).
} 
as BS1 and BS2 and are at 0 and $L$ respectively. Denote by $G(L, \rho, \mathcal{C})$ the subnetwork with length $L$, vehicle density $\rho$ and channel model $\mathcal{C}$. We investigate the access probability $p_{a}$ that an arbitrary vehicle in $G(L, \rho, \mathcal{C})$ can access either BS (either BS1 or BS2). We also investigate the probability $p_{c}$ that all vehicles in $G(L, \rho, \mathcal{C})$ are connected to at least one of the BSs at both ends of the subnetwork.

A vehicle is said to be located at $x$ if its Euclidean distance to BS1 is $x$. The probability that a vehicle located at $x$ is not directly connected to BS1 and BS2 are $1-g_{b}^{\mathcal{C}}(x)$ and $1-g_{b}^{\mathcal{C}}(L-x)$ respectively. Because the event that a vehicle is not directly connected to BS1 and the event that the same vehicle is not directly connected to BS2 are independent, the probability that the vehicle is directly connected to either BS1 or $\mathrm{BS} 2$ is then

$$
p_{1}(x)=1-\left(1-g_{b}^{\mathcal{C}}(x)\right)\left(1-g_{b}^{\mathcal{C}}(L-x)\right) .
$$

In order to derive $p_{a}$ and $p_{c}$ we need the following lemmas.

Lemma 1. Let $K_{1}$ be the set of vehicles in the subnetwork $G(L, \rho, \mathcal{C})$ which are directly connected to either BS1 or $B S 2$, then $K_{1}$ has an inhomogeneous Poisson distribution with density $\rho p_{1}(x)$ where $p_{1}(x)$ is given by $E q$. (2).

Proof: Let $K$ denotes the set of vehicles in $G(L, \rho, \mathcal{C})$. Then $K$ has a homogeneous Poisson distribution with density $\rho$ over the segment $[0, L]$. Consider a realization of $K$ and remove a vehicle located at $x$ from this realization with probability $1-p_{1}(x)$, independent of the removal probability of other vehicles. The remaining set of vehicles can be effectively viewed as a realization of $K_{1}$. Note that the above procedure which removes/retains vehicles independently with some probabilities is called thinning [38]. Let $\mathcal{N}\left(K_{1}\right)$ be the number of vehicles in $K_{1}$. Then following the law of total probability

$$
\begin{aligned}
& \operatorname{Pr}\left(\mathcal{N}\left(K_{1}\right)=j\right) \\
& =\sum_{i=j}^{\infty} \operatorname{Pr}(\mathcal{N}(K)=i) \operatorname{Pr}\left(\mathcal{N}\left(K_{1}\right)=j \mid \mathcal{N}(K)=i\right) .
\end{aligned}
$$

For a randomly chosen vehicle in $K$, the vehicle is known to be uniformly distributed in $[0, L]$. Hence, the probability that a vehicle is in $K_{1}$ given that the vehicle is in $K$ is

$$
q=\frac{1}{L} \int_{0}^{L} p_{1}(x) d x
$$

Since the probability of a randomly chosen vehicle in $K$ being directly connected to either BS1 or BS2 are identically and independently distributed, the probability that among $i$ vehicles in $K$ there are $j$ vehicles in $K_{1}$ follows the binomial distributed $B(i, q)$. Thus

$$
\operatorname{Pr}\left(\mathcal{N}\left(K_{1}\right)=j \mid \mathcal{N}(K)=i\right)=\left(\begin{array}{l}
i \\
j
\end{array}\right) q^{j}(1-q)^{i-j} .
$$

Applying Eq. (1) and (5) into Eq. (3) we have

$$
\begin{aligned}
\operatorname{Pr}\left(\mathcal{N}\left(K_{1}\right)=j\right) & =\sum_{i=j}^{\infty} \frac{(\rho L)^{i}}{i !} e^{-\rho L}\left(\begin{array}{c}
i \\
j
\end{array}\right) q^{j}(1-q)^{i-j} \\
& =\frac{\left(\int_{0}^{L} \rho p_{1}(x) d x\right)^{j}}{j !} e^{-\int_{0}^{L} \rho p_{1}(x) d x} .
\end{aligned}
$$

Furthermore, denote by $\mathcal{N}\left(K_{1}(l)\right)$ the number of vehicles in a road segment $l$ within $[0, L]$ which are directly connected to at least one BSs. Using the above procedure, it is trivial to show that

$$
\operatorname{Pr}\left(\mathcal{N}\left(K_{1}(l)\right)=j\right)=\frac{\left(\int_{x \in l} \rho p_{1}(x) d x\right)^{j}}{j !} e^{-\int_{x \in l} \rho p_{1}(x) d x} .
$$

For $n$ mutually disjoint road segments $l_{1}, l_{2}, \cdots, l_{n}$ in $[0, L]$, the random variables $\mathcal{N}\left(K_{1}\left(l_{1}\right)\right), \cdots, \mathcal{N}\left(K_{1}\left(l_{n}\right)\right)$ are mutually independent. This is because the event that one vehicle is directly connected to either BS1 or BS2 is not affected by the locations of other vehicles, and whether or not those vehicles are directly connected to either BS1 or BS2. Consequently, the existence and locations of vehicles in one road segment will not affect the number of vehicles directly connected to BS1 or BS2 in another disjoint road segment. With the above independence property, Eq. (6) and (7), the proof is then complete. Note that some parts of the proof are similar to the arguments used in [38].

Lemma 2. Let $p_{2}(x)$ be the probability that a vehicle located at $x$ in $G(L, \rho, \mathcal{C})$ is directly connected to at least one vehicle in $K_{1}$, then

$$
p_{2}(x)=1-e^{-\int_{0}^{L} g_{v}^{\mathcal{C}}(\|x-y\|) \rho p_{1}(y) d y}
$$

where $p_{1}(y)$ is given by Eq. (2) and $\|$.$\| denotes the Euclidean$ norm.

Proof: Imagine we partition $[0, L]$ into $L / d y$ nonoverlapping intervals of differential length $d y$. Since $d y$ is a very small value, the probability that there exist more than one vehicle within each interval of length $d y$ can be ignored and the probability that there exists exactly one vehicle within $d y$ is $\rho d y$. The probability that there exists a vehicle in $[y, y+d y]$ which is also in $K_{1}$ is then given by $\rho p_{1}(y) d y$. Note that the vehicles at $x$ and $y$ are directly connected to each other with probability $g_{v}^{\mathcal{C}}(\|x-y\|)$. Therefore, the probability that a vehicle at $x$ is directly connected to a vehicle in $K_{1}$ and is located in $[y, y+d y]$ is $g_{v}^{\mathcal{C}}(\|x-y\|) \rho p_{1}(y) d y$.

Let $h(x, y)$ denotes the probability that the vehicle at $x$ is not directly connected to any of the vehicles in $K_{1}$ located within $[0, y]$. Because the events that distinct pairs of vehicles are directly connected are independent, the event that the vehicle at $x$ is not directly connected to any of the vehicles in $K_{1}$ located within $[0, y]$ is independent of the event that the same vehicle is not directly connected to the vehicle in $K_{1}$ located within $[y, y+d y]$ (if there is any). We have

$$
h(x, y+d y)=h(x, y)\left(1-g_{v}^{\mathcal{C}}(\|x-y\|) \rho p_{1}(y) d y\right)
$$

where the second term on the right hand side of the equation is the complement of the probability that a vehicle at $x$ is directly connected to a vehicle in $K_{1}$ and located in $[y, y+d y]$. Eq. (9) leads to

$$
d h(x, y)=-h(x, y) g_{v}^{\mathcal{C}}(\|x-y\|) \rho p_{1}(y) d y .
$$

Therefore the probability that a vehicle at $x$ is not directly connected to any vehicle in $K_{1}$ is

$$
h(x)=e^{-\int_{0}^{L} g_{v}^{\mathcal{C}}(\|x-y\|) \rho p_{1}(y) d y} .
$$


The result follows immediately.

The following two theorems give the access probability $p_{a}$ and the connectivity probability $p_{c}$ respectively.

Theorem 1. Denote by $p_{a}(x)$ the access probability of a vehicle at $x$, i.e. the probability that the vehicle at $x$ is connected to either BS1 or BS2 in at most two hops. Then

$$
p_{a}(x)=1-\left(1-p_{1}(x)\right)\left(1-p_{2}(x)\right)
$$

where $p_{1}(x)$ is given by Eq. (2) and $p_{2}(x)$ is given by Eq. (8).

Proof: The result follows immediately from the observation that the event that a vehicle at $x$ is directly connected to either BS1 or BS2 is independent of the event that the same vehicle is directly connected to at least one vehicle in $K_{1}$.

Theorem 2 (Approximate result). Denote by $p_{c}$ the connectivity probability of $G(L, \rho, \mathcal{C})$, i.e. the probability that all vehicles in the subnetwork $G(L, \rho, \mathcal{C})$ are connected to either $B S 1$ or BS2 in at most two hops. Assume that the event that a vehicle is connected to either BS1 or BS2 in at most two hops is independent of the event that another vehicle is connected to either BS1 or BS2 in at most two hops. Then

$$
p_{c}=e^{-\int_{0}^{L} \rho\left(1-p_{a}(x)\right) d x}
$$

where $p_{a}(x)$ is given by Eq. (12).

Proof: Let $K_{2}$ be the set of vehicles in $G(L, \rho, \mathcal{C})$ which are connected to either BS1 or BS2 in exactly two hops. Together with the definition of $K_{1}$ in Lemma 1, let $\overline{K_{1}+K_{2}}=K \backslash\left(K_{1}+K_{2}\right)$ be the set of vehicles in $G(L, \rho, \mathcal{C})$ which are not connected to either BS1 or BS2 in at most two hops. Apply the thinning procedure for $K$, i.e. consider a realization of $K$ and remove each vehicle located at $x$ independently from this realization, with probability $p_{a}(x)$. The resulting set of vehicles can be viewed as a realization of $\overline{K_{1}+K_{2}}$ under our assumption that the event that one vehicle is connected to either BS in two hops is independent of the event that another vehicle is connected to either BS in two hops, and the probability that vehicle at $x$ is connected to either BS in two hops is $p_{a}(x)$. Using the same technique as that used in the proof of Lemma 1 , it can be readily shown that $\overline{K_{1}+K_{2}}$ has an inhomogeneous Poisson distribution with density $\rho\left(1-p_{a}(x)\right)$. Then all vehicles $G(L, \rho, \mathcal{C})$ are connected to either BS1 or BS2 in at most two hops if and only if $\mathcal{N}\left(\overline{K_{1}+K_{2}}\right)=0$. The result follows.

Note that Theorem 2 only gives an approximate result for the connectivity probability because of the independence assumption. The following lemma proves, in a way, that the event that a vehicle is connected to either BS1 or BS2 in at most two hops is not independent of the event that another vehicle is connected to either BS1 or BS2 in at most two hops.

Lemma 3. Let $h(x)=1-p_{2}(x)$ be the probability that a vehicle at $x$ is not directly connected to any vehicle in $K_{1}$; let $h\left(x_{1}, x_{2}\right)$ be the probability that two vehicles, at $x_{1}$ and $x_{2}$ respectively, are not directly connected to any vehicle in $K_{1}$. Then, $h\left(x_{1}, x_{2}\right) \geq h\left(x_{1}\right) h\left(x_{2}\right)$.

Proof: Let $h\left(x_{1}, x_{2} ; y\right)$ denotes the probability that two vehicles, at $x_{1}$ and $x_{2}$ respectively, are not directly connected to any vehicle in $K_{1}$ located in $[0, y]$. Using the similar argument in Eq. (9), we have

$$
h\left(x_{1}, x_{2} ; y+d y\right)=h\left(x_{1}, x_{2} ; y\right) k\left(x_{1}, x_{2} ; y\right)
$$

where $k\left(x_{1}, x_{2} ; y\right)=\left(1-g_{v}^{\mathcal{C}}\left(\left\|x_{1}-y\right\|\right)\right)\left(1-g_{v}^{\mathcal{C}}\left(\| x_{2}-\right.\right.$ $y \|)) \rho p_{1}(y) d y+\left(1-\rho p_{1}(y) d y\right)$. The first term on the right hand side of $k\left(x_{1}, x_{2} ; y\right)$ is the probability there is a vehicle in $K_{1}$ located in $[y, y+d y]$ and both vehicles in $x_{1}$ and $x_{2}$ are not directly connected to it. The second term on the right hand side of $k\left(x_{1}, x_{2} ; y\right)$ is the probability that there is no vehicle in $K_{1}$ located in $[y, y+d y]$. Expanding the right hand side of $k\left(x_{1}, x_{2} ; y\right)$ we have

$$
\begin{aligned}
k\left(x_{1}, x_{2} ; y\right)= & 1-g_{v}^{\mathcal{C}}\left(\left\|x_{1}-y\right\|\right) \rho p_{1}(y) d y \\
& -g_{v}^{\mathcal{C}}\left(\left\|x_{2}-y\right\|\right) \rho p_{1}(y) d y \\
& +g_{v}^{\mathcal{C}}\left(\left\|x_{1}-y\right\|\right) g_{v}^{\mathcal{C}}\left(\left\|x_{2}-y\right\|\right) \rho p_{1}(y) d y
\end{aligned}
$$

Using the same approach in Lemma 2 we obtain

$$
\begin{aligned}
h\left(x_{1}, x_{2}\right)= & e^{-\int_{0}^{L}\left[g_{v}^{\mathcal{C}}\left(\left\|x_{1}-y\right\|\right)+g_{v}^{\mathcal{C}}\left(\left\|x_{2}-y\right\|\right)\right] \rho p_{1}(y) d y} \\
& \times e^{\int_{0}^{L} g_{v}^{\mathcal{C}}\left(\left\|x_{1}-y\right\|\right) g_{v}^{\mathcal{C}}\left(\left\|x_{2}-y\right\|\right) \rho p_{1}(y) d y} \\
\geq & e^{-\int_{0}^{L}\left[g_{v}^{\mathcal{C}}\left(\left\|x_{1}-y\right\|\right)+g_{v}^{\mathcal{C}}\left(\left\|x_{2}-y\right\|\right)\right] \rho p_{1}(y) d y} \\
= & h\left(x_{1}\right) h\left(x_{2}\right) \quad \text { (from Eq. (11)) }
\end{aligned}
$$

Before obtaining the exact result of the connectivity probability, we introduce some properties in the following lemma.

Lemma 4. Let $p_{c}(\mathbf{y})$ be the connectivity probability of $G(L, \rho, \mathcal{C})$ conditioned on that the number of vehicles directly connected to either $B S$ is $n$ and they are located at $\mathbf{y}=\left\{y_{1}, y_{2}, \cdots, y_{n}: 0 \leq y_{i} \leq L, 1 \leq i \leq n\right\} ;$ let $p_{Y}(\mathbf{y})$ be the probability density function ( $p d f)$ of $\mathbf{y}$ conditioned on that there are $n$ vehicles directly connected to either BS. The following properties hold.

(i) $p_{Y}(\mathbf{y})=\prod_{i=1}^{n} \frac{p_{1}\left(y_{i}\right)}{\int_{0}^{L} p_{1}(x) d x}$

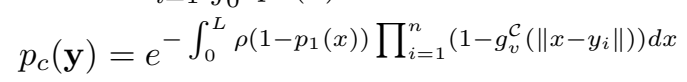

Proof: For $n=1, p_{Y}\left(y_{1}\right)=\frac{p_{1}\left(y_{1}\right)}{\int_{0}^{L} p_{1}(x) d x}$ is the probability that a vehicle in $K_{1}$ is located at $y_{1}$. Since $p_{1}\left(y_{i}\right)$ and $p_{1}\left(y_{j}\right)$ are mutually independent for $i \neq j$, the result follows for Eq. (15).

For Eq. (16), note that a vehicle at $x$ is not connected to any BSs in at most two hops if it is not directly connected to any BSs (the probability is $1-p_{1}(x)$ ) and it is not directly connected to vehicles which are located at $\mathbf{y}$ given that these vehicles are in $K_{1}$ (the probability is $1-g_{v}^{\mathcal{C}}\left(\left\|x-y_{i}\right\|\right)$ for $1 \leq i \leq n)$. That is, vehicle at $x$ cannot access any BS in at most two hops with probability

$$
\left(1-p_{1}(x)\right) \prod_{i=1}^{n}\left(1-g_{v}^{\mathcal{C}}\left(\left\|x-y_{i}\right\|\right)\right) .
$$

Eq. (17) is valid when $x \notin \mathbf{y}$. When $x=y_{j}$ for arbitrary $j$, we assume that $g_{v}^{\mathcal{C}}(0)=1$. This implies that $p_{a}(x \mid \mathbf{y})=1$. So Eq. (17) is still valid when $x \in \mathbf{y}$. Applying the thinning 
procedure and the technique used in Lemma 1, we have the number of vehicles which are neither directly connected to any BSs nor directly connected to any of the vehicles at $\mathbf{y}$ is an inhomogeneous Poisson random variable with density $\rho\left(1-p_{1}(x)\right) \prod_{i=1}^{n}\left(1-g_{v}^{\mathcal{C}}\left(\left\|x-y_{i}\right\|\right)\right)$. The result follows immediately.

Theorem 3 (Exact result). Denote by $p_{c}$ the connectivity probability of $G(L, \rho, \mathcal{C})$, i.e. the probability that all vehicle in the subnetwork $G(L, \rho, \mathcal{C})$ is connected to either BS1 or BS2 in at most two hops. Then

$$
p_{c}=\sum_{n=0}^{\infty} \operatorname{Pr}\left(\mathcal{N}\left(K_{1}\right)=n\right)\left[\int_{[0, L]^{n}} p_{c}(\mathbf{y}) p_{Y}(\mathbf{y}) d \mathbf{y}\right]
$$

where $p_{c}(\mathbf{y})$ and $p_{Y}(\mathbf{y})$ are given by Lemma $4 ; \operatorname{Pr}\left(\mathcal{N}\left(K_{1}\right)=\right.$ $n$ ) is given by Lemma 1 . When $n=0$, we declare

$$
\begin{aligned}
& \left.\int_{[0, L]^{n}} p_{c}(\mathbf{y}) p_{Y}(\mathbf{y}) d \mathbf{y}\right|_{n=0}=\left.p_{c}(\mathbf{y}) p_{Y}(\mathbf{y})\right|_{n=0} \\
& =e^{-\int_{0}^{L} \rho\left(1-p_{1}(x)\right) d x} .
\end{aligned}
$$

Proof: Eq. (18) directly follows from the law of total probability, so the details are omitted here.

Eq. (18) gives an exact formula for the connectivity probability which does not rely on the assumption that the event that a vehicle is connected to either BS in two hops and the event that another vehicle is connected to either $\mathrm{BS}$ in two hops are independent. However Eq. (18) is much more complicated than the approximate result in Eq. (13). In many situations, Eq. (13) provides a reasonably accurate result for the connectivity probability. Therefore we include both results in this paper.

\section{Performance Evaluation under Specific CHANNEL MODELS}

Based on the analysis in Section IV, we further derive and compare in this section the access probability and connectivity probability performance under two specific channel models, i.e. unit disk model and log-normal shadowing model.

\section{A. Unit Disk Model}

In the unit disk model $\mathcal{U}$, assume that two vehicles are directly connected if and only if their Euclidean distance is less than or equal to $r$; assume that a vehicle and a BS are directly connected if and only if their Euclidean distance is not more than $R$. In other words,

$$
g_{v}^{\mathcal{U}}(x)=\left\{\begin{array}{ll}
1 & \text { if } x \leq r \\
0 & \text { otherwise, }
\end{array} \quad g_{b}^{\mathcal{U}}(x)= \begin{cases}1 & \text { if } x \leq R \\
0 & \text { otherwise. }\end{cases}\right.
$$

where $r$ and $R$ are predetermined values, commonly known as the transmission ranges. Typically we have $R>r$. Applying the above equations into Eq. (2), (8) and (12) we obtain the access probability under the unit disk model $\mathcal{U}$ :

(I) For $0<L \leq 2 R$,

we have $p_{1}(x)=1$ implies that $p_{a}(x)=1$ for $x \in$ $[0, L]$. Hence,

$$
p_{a}=1
$$

(II) For $2 R<L \leq 2 R+r$, we have $p_{1}(x)$ is 0 when $x \in(R, L-R)$, and 1 otherwise. When $x \in(R, L-R)$, Eq. (8) becomes

$$
\begin{aligned}
p_{2}(x) & =1-e^{-\int_{0}^{R} g_{v}^{\mathcal{C}}(\|x-y\|) \rho d y-\int_{L-R}^{L} g_{v}^{\mathcal{C}}(\|x-y\|) \rho d y} \\
& =1-e^{-\int_{x-r}^{R} \rho d y-\int_{L-R}^{x+r} \rho d y} \\
& =1-e^{-\rho(2 R+2 r-L)} .
\end{aligned}
$$

So substitute $p_{2}(x)$ into Eq. (12),

$$
\begin{aligned}
p_{a} & =\frac{2 R}{L}+\frac{L-2 R}{L}\left(1-e^{-\rho(2 R+2 r-L)}\right) \\
& =1-\frac{L-2 R}{L} e^{-\rho(2 R+2 r-L)} .
\end{aligned}
$$

(III) For $2 R+r<L \leq 2 R+2 r$, we have for $x \in(R, L-R)$, Eq. (8) becomes

$$
\begin{aligned}
p_{2}(x) & =1-e^{-\int_{0}^{R} g_{v}^{\mathcal{C}}(\|x-y\|) \rho d y-\int_{L-R}^{L} g_{v}^{\mathcal{C}}(\|x-y\|) \rho d y} \\
& =1-e^{-\int_{x-r}^{R} \rho d y-\int_{L-R}^{x+r} \rho d y} .
\end{aligned}
$$

So substitute $p_{2}(x)$ into Eq. (12),

$$
\begin{aligned}
p_{a}= & \frac{2 R}{L}+\frac{1}{L} \int_{R}^{L-R-r}\left(1-e^{-\rho(R+r-x)}\right) d x \\
& +\frac{1}{L} \int_{L-R-r}^{R+r}\left(1-e^{-\rho(2 R+2 r-L)}\right) d x \\
& +\frac{1}{L} \int_{R+r}^{L-R}\left(1-e^{-\rho(R+r+x-L)}\right) d x \\
= & 1+\frac{2}{\rho L}\left(e^{-\rho r}-e^{-\rho(2 R+2 r-L)}\right) \\
& -\frac{2 R+2 r-L}{L} e^{-\rho(2 R+2 r-L)}
\end{aligned}
$$

(IV) For $L>2 R+2 r$,

From Eq. (8)

$$
p_{2}(x)=\left\{\begin{aligned}
1-e^{-} \int_{x-r}^{R} \rho d y & =1-e^{-\rho(R+r-x)} \\
& \text { when } x \in(R, R+r], \\
1-e^{-\int_{L-R}^{x+r} \rho d y}=1-e^{-\rho(R+r+x-L)} & \\
& \text { when } x \in[L-R-r, L-R), \\
0 \quad & \text { when } x \in(R+r, L-R-r) .
\end{aligned}\right.
$$

So substituting $p_{2}(x)$ into Eq. (12),

$$
\begin{aligned}
p_{a}= & \frac{2 R}{L}+\frac{1}{L} \int_{R}^{R+r}\left(1-e^{-\rho(R+r-x)}\right) d x \\
& +\frac{1}{L} \int_{L-R-r}^{L-R}\left(1-e^{-\rho(R+r+x-L)}\right) d x \\
= & \frac{2 R+2 r}{L}+\frac{2\left(e^{-\rho r}-1\right)}{\rho L} .
\end{aligned}
$$

To derive the equations for the connectivity probability (exact result), we first look at Lemma 4. For the unit disk model, $p_{1}(x)$ is 1 when $x \in[0, R] \cup[L-R, L]$ and zero otherwise. Hence, Eq. (15) becomes

$$
p_{Y}(\mathbf{y})=\frac{1}{(\min (2 R, L))^{n}}
$$


when $y_{i} \in[0, R] \cup[L-R, L], \forall y_{i}$, and zero otherwise. Eq. (16) becomes

$$
p_{c}(\mathbf{y})=e^{-\int_{R}^{L-R} \rho \prod_{i=1}^{n}\left(1-g_{v}^{\mathcal{C}}\left(\left\|x-y_{i}\right\|\right)\right) d x} .
$$

Note that $\prod_{i=1}^{n}\left(1-g_{v}^{\mathcal{C}}\left(\left\|x-y_{i}\right\|\right)\right)$ is 1 when $\left\|x-y_{i}\right\|>r$ for all $y_{i}$. For $L \leq 2 R$, we can easily obtain $p_{c}$ from Eq. (18) by substituting Eq. (19) and (20) into it (will be shown later). To obtain the result for $L>2 R$, the following transformation will simplify the arithmetic work.

Let $S_{a}$ (and $S_{b}$ ) be the set of vehicles in $[0, R]$ (and $[L-$ $R, L]$ ) which, by definition, are also in $K_{1}$. Let $\mathcal{N}\left(S_{a}\right)$ (and $\mathcal{N}\left(S_{b}\right)$ ) be the number of vehicles in $S_{a}$ (and $S_{b}$ ). Note that $S_{a} \cup S_{b}=K_{1}$ and $\mathcal{N}\left(S_{a}\right)+\mathcal{N}\left(S_{b}\right)=\mathcal{N}\left(K_{1}\right)$. Let $y_{a}$ (and $\left.y_{b}\right)$ be the location of the vehicle in $S_{a}$ (and $S_{b}$ ) which is furthest from BS1 (and BS2). That is,

$$
\begin{aligned}
y_{a} & = \begin{cases}0 & \text { if } S_{a}=\emptyset \\
\max \left\{y: y \in S_{a}\right\} & \text { otherwise }\end{cases} \\
y_{b} & = \begin{cases}L & \text { if } S_{b}=\emptyset \\
\min \left\{y: y \in S_{b}\right\} & \text { otherwise }\end{cases}
\end{aligned}
$$

Therefore, the cumulative probability function of $y_{a}$ is

$$
\begin{aligned}
\operatorname{Pr}\left(y_{a} \leq y_{\text {max }}\right) & =\operatorname{Pr}\left(y_{i} \leq y_{\text {max }}, \forall y_{i} \in S_{a}\right) \\
& =\left(\frac{y_{\text {max }}}{R}\right)^{n_{a}} \quad \text { for } n_{a}=\mathcal{N}\left(S_{a}\right) \geq 1 .
\end{aligned}
$$

With Eq. (21) defines $y_{a}=0$ when $\mathcal{N}\left(S_{a}\right)=0$, we have the pdf of $y_{a}$ as

$$
f_{a}\left(y_{a} ; n_{a}\right)= \begin{cases}\frac{n_{a}}{R}\left(\frac{y_{a}}{R}\right)^{n_{a}-1} & \text { if } n_{a} \geq 1 \\ \delta\left(y_{a}\right) & \text { if } n_{a}=0 .\end{cases}
$$

Similarly we have the pdf of $y_{b}$ as

$$
f_{b}\left(y_{b} ; n_{b}\right)= \begin{cases}\frac{n_{b}}{R}\left(\frac{L-y_{b}}{R}\right)^{n_{b}-1} & \text { if } n_{b} \geq 1 \\ \delta\left(L-y_{b}\right) & \text { if } n_{b}=0\end{cases}
$$

With $y_{a}$ and $y_{b}$, we can rewrite Eq. (20) into

$$
p_{c}\left(y_{a}, y_{b}\right)=e^{-\int_{\max \left\{R, y_{a}+r\right\}}^{\min \left\{L-R, y_{b}-r\right\}} \rho d x}
$$

and Eq. (18) can be transformed into

$$
\begin{aligned}
p_{c}= & \sum_{n_{a}=0}^{\infty} \sum_{n_{b}=0}^{\infty} \operatorname{Pr}\left(\mathcal{N}\left(S_{a}\right)=n_{a}\right) \operatorname{Pr}\left(\mathcal{N}\left(S_{b}\right)=n_{b}\right) \\
& {\left[\int_{0}^{R} \int_{L-R}^{L} p_{c}\left(y_{a}, y_{b}\right) f_{a}\left(y_{a} ; n_{a}\right) f_{b}\left(y_{b} ; n_{b}\right) d y_{b} d y_{a}\right] }
\end{aligned}
$$

for $L>2 R$. Eq. (24) can be further simplified under different cases. For $n_{a}>0$ and $n_{b}>0$, Eq. (24) becomes

$$
\begin{aligned}
p_{c}^{\left(n_{a}>0, n_{b}>0\right)} & \int_{0}^{R} \int_{L-R}^{L} p_{c}\left(y_{a}, y_{b}\right)\left[\sum_{n_{a}=1}^{\infty} \sum_{n_{b}=1}^{\infty} \operatorname{Pr}\left(\mathcal{N}\left(S_{a}\right)=n_{a}\right)\right. \\
& \left.\operatorname{Pr}\left(\mathcal{N}\left(S_{b}\right)=n_{b}\right) f_{a}\left(y_{a} ; n_{a}\right) f_{b}\left(y_{b} ; n_{b}\right)\right] d y_{b} d y_{a} \\
= & \int_{0}^{R} \int_{L-R}^{L} p_{c}\left(y_{a}, y_{b}\right)\left[\sum_{n_{a}=1}^{\infty} \sum_{n_{b}=1}^{\infty} \frac{(\rho R)^{n_{a}}}{n_{a} !} e^{-\rho R}\right. \\
& \left.\frac{(\rho R)^{n_{b}}}{n_{b} !} e^{-\rho R} \frac{n_{a}}{R}\left(\frac{y_{a}}{R}\right)^{n_{a}-1} \frac{n_{b}}{R}\left(\frac{L-y_{b}}{R}\right)^{n_{b}-1}\right] d y_{b} d y_{a} \\
= & \int_{0}^{R} \int_{L-R}^{L} p_{c}\left(y_{a}, y_{b}\right) \rho^{2} e^{-2 \rho R} \\
& {\left[\sum_{n_{a}=1}^{\infty} \frac{\left(\rho y_{a}\right)^{n_{a}-1}}{\left(n_{a}-1\right) !}\right]\left[\sum_{n_{b}=1}^{\infty} \frac{\left(\rho\left(L-y_{b}\right)\right)^{n_{b}-1}}{\left(n_{b}-1\right) !}\right] d y_{b} d y_{a} } \\
= & \int_{0}^{R} \int_{L-R}^{L} p_{c}\left(y_{a}, y_{b}\right) \rho^{2} e^{-2 \rho R} e^{\rho y_{a}} e^{\rho\left(L-y_{b}\right)} d y_{b} d y_{a} .
\end{aligned}
$$

For $n_{a}=0$ and $n_{b}>0$, Eq. (24) becomes

$$
\begin{aligned}
& p_{c}^{\left(n_{a}=0, n_{b}>0\right)} \\
& =\sum_{n_{b}=1}^{\infty} e^{-\rho R} \frac{(\rho R)^{n_{b}}}{n_{b} !} e^{-\rho R}\left[\int_{L-R}^{L} p_{c}\left(0, y_{b}\right) \frac{n_{b}}{R}\left(\frac{L-y_{b}}{R}\right)^{n_{b}-1} d y_{b}\right] \\
& =\int_{L-R}^{L} p_{c}\left(0, y_{b}\right) \rho e^{-2 \rho R} \sum_{n_{b}=1}^{\infty} \frac{\left(\rho\left(L-y_{b}\right)\right)^{n_{b}-1}}{\left(n_{b}-1\right) !} d y_{b} \\
& =\int_{L-R}^{L} p_{c}\left(0, y_{b}\right) \rho e^{-2 \rho R} e^{\rho\left(L-y_{b}\right)} d y_{b} .
\end{aligned}
$$

With similar steps (omit here) we can obtain for $n_{a}>0$ and $n_{b}=0$, Eq. (24) becomes

$$
p_{c}^{\left(n_{a}>0, n_{b}=0\right)}=\int_{0}^{R} p_{c}\left(y_{a}, L\right) \rho e^{-2 \rho R} e^{\rho y_{a}} d y_{a} .
$$

Note that it can be shown that Eq. (27) equals to (26) by letting $y_{b}=L-y_{a}$, then

$$
\begin{aligned}
p_{c}^{\left(n_{a}>0, n_{b}=0\right)} & =-\int_{L}^{L-R} p_{c}\left(L-y_{b}, L\right) \rho e^{-2 \rho R} e^{\rho\left(L-y_{b}\right)} d y_{b} \\
& =\int_{L-R}^{L} p_{c}\left(0, y_{b}\right) \rho e^{-2 \rho R} e^{\rho\left(L-y_{b}\right)} d y_{b}
\end{aligned}
$$

where $p_{c}\left(L-y_{b}, L\right)=p_{c}\left(0, y_{b}\right)$. Finally for $n_{a}=0$ and $n_{b}=0$,

$$
\begin{aligned}
p_{c}^{\left(n_{a}=0, n_{b}=0\right)} & =e^{-\rho R} e^{-\rho R} p_{c}(0, L)=e^{-2 \rho R} e^{-\int_{R}^{L-R} \rho d x} \\
& =e^{-2 \rho R} e^{-\rho(L-2 R)}=e^{-\rho L} .
\end{aligned}
$$

Using Eq. (25), (26), (27) and (28), we can obtain the connectivity probability as follows. Due to the lengthy (but straightforward) steps involved to derive the results, we omit the intermediate steps and only include the results of Eq. (25) and (26) for readers' convenience.

(I) For $0<L \leq 2 R$, $p_{Y}(\mathbf{y})=\frac{1}{L^{n}}$ from Eq. (15) and $p_{c}(\mathbf{y})=1$ from Eq. (16) 
implies that

$$
p_{c}=\sum_{n=0}^{\infty} \operatorname{Pr}\left(\mathcal{N}\left(K_{1}\right)=n\right)=1 .
$$

(II) For $2 R<L \leq 2 R+r$,

$$
\begin{aligned}
p_{c}^{\left(n_{a}>0, n_{b}>0\right)}= & 1+e^{-\rho L}-2 e^{-\rho R}+e^{-\rho(3 R+r-L)} \\
& +\left(-\frac{1}{4}-\frac{1}{2} \rho(L-2 R)\right) e^{-\rho(2 R+2 r-L)} \\
& -e^{-\rho(L+r-R)}+\frac{1}{4} e^{-\rho(L+2 r-2 R)}, \\
p_{c}^{\left(n_{a}=0, n_{b}>0\right)}= & -e^{-\rho L}+e^{-\rho R}-\frac{1}{2} e^{-\rho(3 R+r-L)} \\
& +\frac{1}{2} e^{\rho(L+r-R)}, \\
p_{c}= & 1+\frac{1}{4} e^{-\rho(L+2 r-2 R)} \\
& +\left(-\frac{1}{4}-\frac{1}{2} \rho(L-2 R)\right) e^{-\rho(2 R+2 r-L)} .
\end{aligned}
$$

(III) For $2 R+r<L \leq 2 R+2 r$,

$$
\begin{array}{rl}
p_{c}^{\left(n_{a}>0, n_{b}>0\right)} & \\
= & 1+e^{-\rho L}+\frac{1}{2} e^{-\rho(L-2 R)}-e^{-\rho(L+r-R)} \\
& +\left(-\frac{3}{4}-\frac{1}{2} \rho(2 R+2 r-L)\right) e^{-\rho(2 R+2 r-L)} \\
& +\frac{1}{4} e^{-\rho(L+2 r-2 R)}-e^{-\rho(L-R-r)}, \\
\left.p_{c}^{\left(n_{a}\right.}=0, n_{b}>0\right) & \\
= & -e^{-\rho L}+\frac{1}{2} e^{-\rho(L+r-R)}+\frac{1}{2} e^{-\rho(L-R-r)}, \\
p_{c} & 1+\frac{1}{2} e^{-\rho(L-2 R)}+\frac{1}{4} e^{-\rho(L+2 r-2 R)} \\
& +\left(-\frac{3}{4}-\frac{1}{2} \rho(2 R+2 r-L)\right) e^{-\rho(2 R+2 r-L)} .
\end{array}
$$

(IV) For $L>2 R+2 r$,

$$
\begin{aligned}
& p_{c}^{\left(n_{a}>0, n_{b}>0\right)} \\
& =e^{-\rho L}+\frac{1}{4} e^{-\rho(L+2 r-2 R)}-e^{-\rho(L+r-R)} \\
& +\frac{1}{4} e^{-\rho(L-2 R-2 r)}-e^{-\rho(L-R-r)}+\frac{1}{2} e^{-\rho(L-2 R)}, \\
& p_{c}^{\left(n_{a}=0, n_{b}>0\right)} \\
& =-e^{-\rho L}+\frac{1}{2} e^{-\rho(L+r-R)}+\frac{1}{2} e^{-\rho(L-R-r)}, \\
p_{c}= & \frac{1}{4} e^{-\rho(L+2 r-2 R)}+\frac{1}{4} e^{-\rho(L-2 R-2 r)}+\frac{1}{2} e^{-\rho(L-2 R)} .
\end{aligned}
$$

\section{B. Log-normal Shadowing Model}

The log-normal model $\mathcal{L}$ is commonly used to model the real world signal propagation where the transmit power loss increases logarithmically with the Euclidean distance between two wireless nodes and varies log-normally due to the shadowing effect caused by surrounding environment. In the log-normal model, we formulate the received power (in dB) at a destination vehicle as

$$
p_{r x}=p_{0}-10 \alpha \log _{10} \frac{l}{d_{0}}+N_{\sigma}
$$

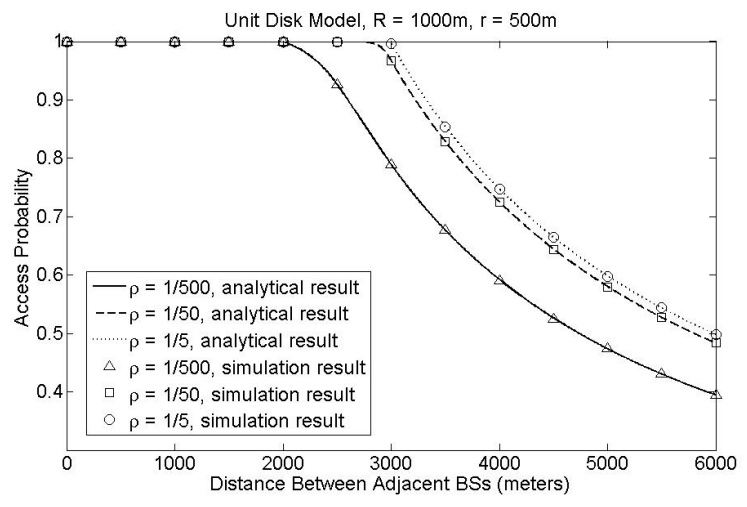

Fig. 3. Access probability with $L$ changing under the unit disk model, $R=1000 \mathrm{~m}, r=500 \mathrm{~m}, \rho=1 / 5,1 / 50,1 / 500$ vehicles $/ \mathrm{m}$ respectively.

where $p_{r x}$ is the received power (in $\mathrm{dBmW}$ ) at the destination vehicle; $p_{0}$ is the power (in $\mathrm{dBmW}$ ) at a reference distance $d_{0}$; $\alpha$ is the path loss exponent; $N_{\sigma}$ is a Gaussian random variable with zero mean and variance $\sigma^{2} ; l$ is the Euclidean distance between the two vehicles (or a vehicle and a BS depending on the context). A source vehicle can establish a direct connection to a destination vehicle if the received power at the destination vehicle $p_{r x}$ is greater than or equal to a certain threshold power $p_{t h}^{v}$. Similarly, a source vehicle can establish a two-way direct connection to a destination $\mathrm{BS}$ if the received power at the destination $\mathrm{BS} p_{r x}$ is greater than or equal to a certain threshold power $p_{t h}^{b}$. In this paper, we assume that wireless connections between vehicles, and between vehicles and BSs, are symmetric. Note that when $\sigma=0$, the log-normal model reduces to the unit disk model. Due to this fact, we assign $p_{t h}^{v}=p_{0}-10 \alpha \log _{10} \frac{r}{d_{0}}, p_{t h}^{b}=p_{0}-10 \alpha \log _{10} \frac{R}{d_{0}}$ so that the results under log-normal model can be compared with the results under the unit disk model later. It can be shown that under the log-normal model

$$
g_{v}^{\mathcal{L}}(x)=\operatorname{Pr}\left(p_{r x} \geq p_{t h}^{v}\right)=Q\left(\frac{10 \alpha}{\sigma} \log _{10} \frac{x}{r}\right) .
$$

where function $Q(y)=\frac{1}{\sqrt{2 \pi}} \int_{y}^{\infty} e^{-\frac{x^{2}}{2}} d x$ is the tail probability of the standard normal distribution. Similarly, $g_{b}^{\mathcal{L}}(x)=$ $Q\left(\frac{10 \alpha}{\sigma} \log _{10} \frac{x}{R}\right)$. When $\sigma=0, g_{v}^{\mathcal{L}}(x)=\operatorname{Pr}(x \leq r)$, $g_{b}^{\mathcal{L}}(x)=\operatorname{Pr}(x \leq R)$ and the log-normal model becomes the unit disk model as expected.

The access probability can then be obtained for different values of $\alpha$ and $\sigma$ by computing Eq. (12) using any numerical integration technique. The approximate and exact results for the connectivity probability can be obtained by computing Eq. (13) and (18) using any numerical integration technique.

\section{AnAlytical and Simulation Results}

\section{A. Unit Disk Model}

Fig. 3 shows the access probability given different values of $L$ and $\rho$. The analytical results are verified by the simulation results which are obtained from 40000 randomly generated network topologies. As the number of instances of random networks used in the simulation is very large, the confidence interval is too small to be distinguishable and hence ignored in this plot as well as other plots. As shown in the figure, the access probability decreases with $L$ when $L$ exceeds some 


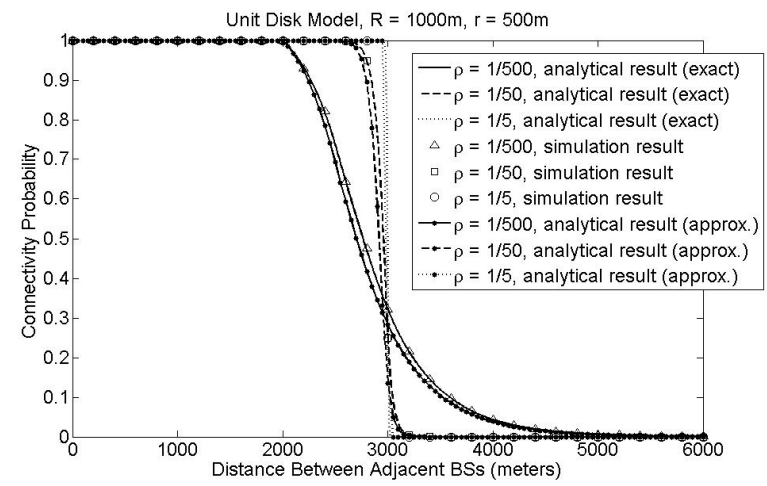

Fig. 4. Connectivity probability with $L$ changing under the unit disk model, $R=1000 \mathrm{~m}, r=500 \mathrm{~m}, \rho=1 / 5,1 / 50,1 / 500$ vehicles $/ \mathrm{m}$ respectively.

limits. For small $\rho$, the access probability decreases as soon as $L>2 R$. That is because when the vehicle density $\rho$ (number of vehicles per meter) is low, a vehicle is either directly connected to a BS or disconnected, i.e cannot reach any BS in at most two hops. It is hard for the vehicle to find a one-hop relay in its range via which it can access a BS if it is not within the transmission range of any BS. However for large $\rho$, it is easier for the vehicle, which is not within the transmission range of any BS, to find a one-hop relay to access the BS. In general the access probability increases with an increase in $\rho$, and the reason is that when the vehicle density increases, the probability increases for vehicles in the gap of the transmission ranges of BSs to find a neighbor within the transmission range of a BS to act as a relay.

Similarly, Fig. 4 shows the connectivity probability for different values of $L$ and $\rho$. The exact analytical results are verified by the simulation results. The approximate analytical result is shown to be reasonably close to the exact analytical result. The figure shows that when $L \leq 2 R+r=2500$ meters, it is easy for all vehicles to be connected to either BS in at most two hops, hence the connectivity probability is high. As $L$ gets larger, it is harder for all vehicles to be connected to the BSs due to the larger possible distances between the vehicles and the BSs. This causes a drop in the connectivity probability, and the connectivity probability tends to zero as $L$ goes to infinity. The transition of the connectivity probability from 1 to 0 gets sharper as the vehicle density increases. As $\rho$ goes to infinity, the transition happens at the critical distance $L=2 R+2 r=3000$ meters, below which the network is disconnected with a high probability and above which the network is connected with a high probability. Furthermore, the networks with a larger $\rho$ have a higher connectivity probability than the networks with a smaller $\rho$ when $L$ is small. This is because when the vehicle density is large, it is easier for vehicles not directly connected to a BS to find a vehicle within its communication range and is directly connected to a BS to act as a relay. When $L$ is large, the networks with a larger $\rho$ have a lower connectivity probability than the networks with a smaller $\rho$. This is because at large values of $L$ when the vehicle density is large it is easier to have at least one vehicle which is located too far from the BSs to be connected to a BS in at most two hops.

Fig. 5 shows how the transmission range of the vehicles $r$ affect the access probability. It shows that the access

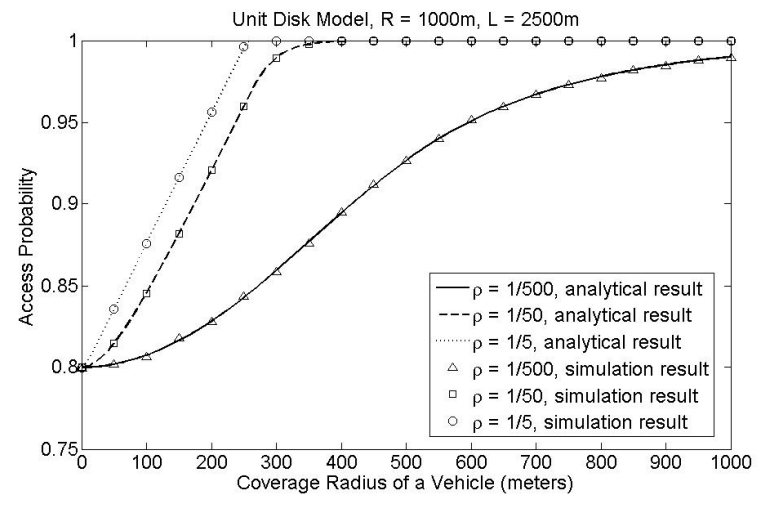

Fig. 5. Access probability with $r$ changing under the unit disk model, $R=1000 \mathrm{~m}, L=2500 \mathrm{~m}, \rho=1 / 5,1 / 50,1 / 500$ vehicles $/ \mathrm{m}$ respectively.

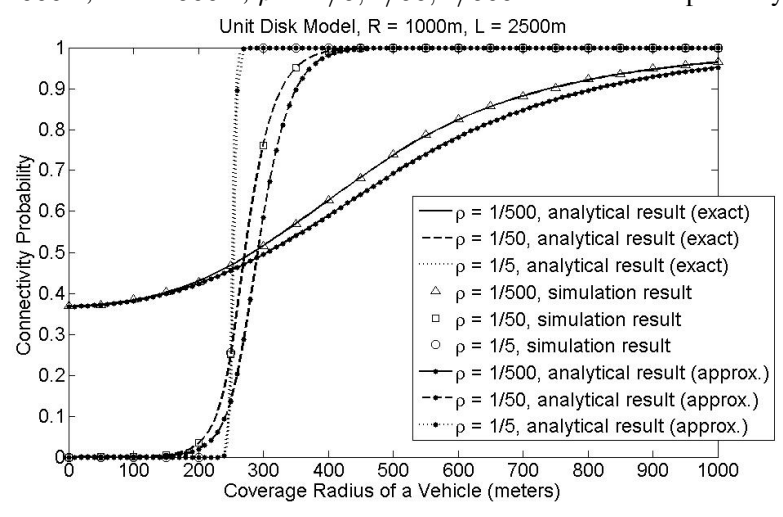

Fig. 6. Connectivity probability with $r$ changing under the unit disk model, $R=1000 \mathrm{~m}, L=2500 \mathrm{~m}, \rho=1 / 5,1 / 50,1 / 500$ vehicles $/ \mathrm{m}$ respectively.

probability increases with $r$, and when $\rho$ is large enough, the access probability could be quite close to 1 . And it shows again that the access probability increases with an increase in $\rho$.

With a similar setup, Fig. 6 shows the sensitivity of the connectivity probability to $r$. For a large $\rho$, around a certain value of $r$ a small increase in $r$ will incur a dramatic increase in the connectivity probability from near 0 to near 1 , i.e. the well-known phase transition phenomenon. From the figure it shows that such phenomenon does not exist for small $\rho$. Fig. 6 also shows a scenario where there may be a significant gap between the approximate and exact results for connectivity probability.

Fig. 7 supported our conclusion that an increase in $\rho$ will improve the access probability as it shows that the access probability monotonically increases with $\rho$. While $\rho$ is relatively small, and the width of the gap region not directly covered by any of the BSs is relatively large, the access probability will be low, and thus, in this circumstance, network operator should consider to deploy more BSs along the highway for better connectivity and greater access probability.

\section{B. Log-normal Shadowing Model}

Fig. 8 shows the access probability under the log-normal shadowing model. In general, it is easier for the vehicles in the subnetwork to get access to any BS under the log-normal model. As $\sigma$ increases, the access probability improves. The improvement in access probability is more significant for high vehicular density. 


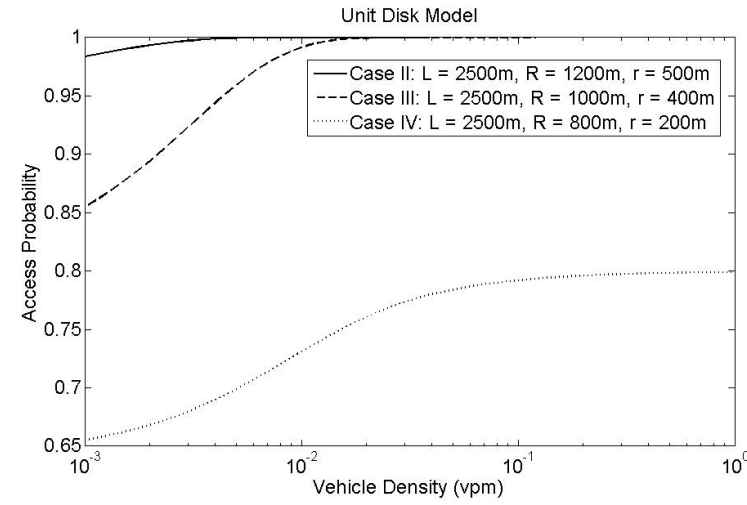

Fig. 7. Access probability with $\rho$ changing under the unit disk model, $L$, $R, r$ are constants.

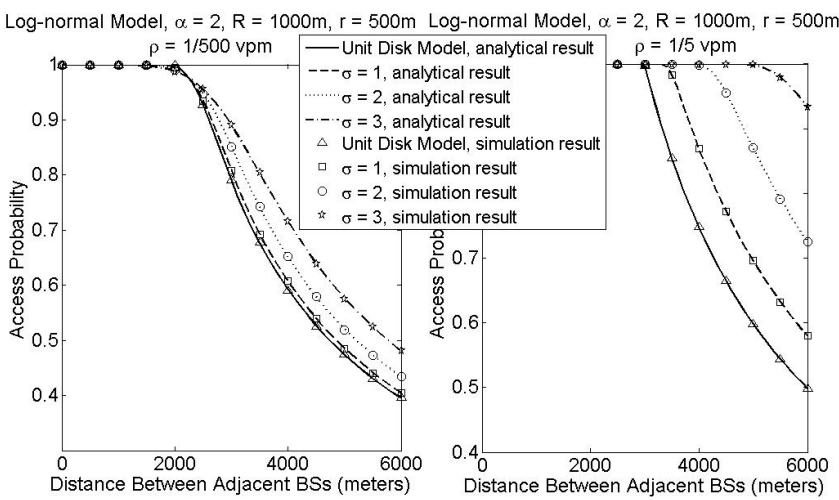

Fig. 8. Access probability with $L$ changing under the log-normal model, $R=1000 \mathrm{~m}, r=500 \mathrm{~m}, \rho=1 / 5,1 / 500$ vehicles/m respectively under different values of $\sigma . R(r)$ is the transmission range of a BS (vehicle) ignoring shadowing effect, i.e. $\sigma=0$.

Fig. 9 shows the connectivity probability under the lognormal model when the vehicle density is low $\left(\rho=\frac{1}{500} \mathrm{vpm}\right)$. The exact analytical results are verified by the simulation results. As the vehicle density increases, the computational complexity involved in numerically computing the exact result increases very quickly. As such, we only provide the exact analytical results for low vehicle density. Furthermore, Fig. 9 shows that the approximate analytical results are reasonably close to the true values when the vehicle density is low. However, as shown in Fig. 10, the discrepancy between the approximate results and the true values can be significant when the vehicle density is high $\left(\rho=\frac{1}{50}, \frac{1}{5} \mathrm{vpm}\right)$. In general, the approximate analytical result always under-estimate the simulation result. Same situation can be observed for the result under the unit disk model. This can be explained by Lemma 3 that a vehicle is more likely to be able to access to any BS where there is another vehicle nearby that can access to the BSs. Because of the independence assumption used in obtaining the approximate analytical result, the approximate result will under-estimate the true value.

\section{CONCLUSIONS}

In this paper, we analyzed the connectivity probability and the access probability for a given network bounded by two adjacent base stations, and vehicles in the network are Poissonly distributed with known density and each vehicle can communicate with a base station in at most two hops. Under

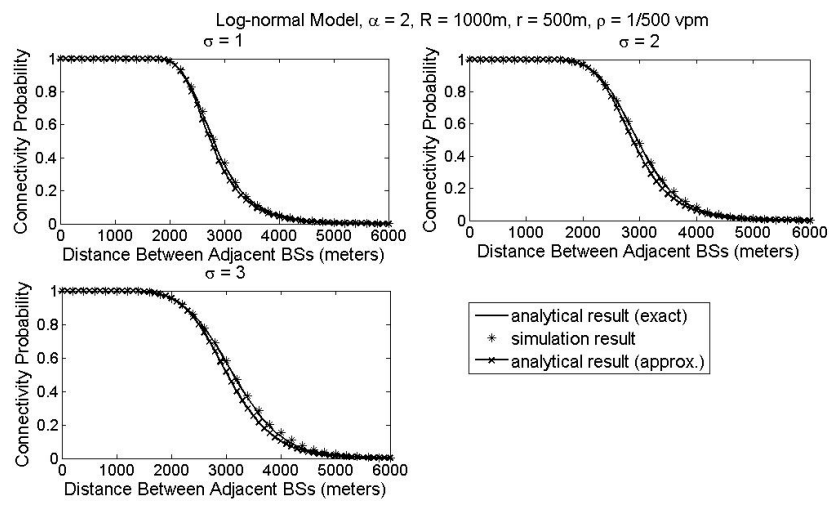

Fig. 9. Connectivity probability with $L$ changing under the log-normal model, $R=1000 \mathrm{~m}, r=500 \mathrm{~m}, \rho=1 / 500$ vehicles $/ \mathrm{m}$ under different values of $\sigma . R(r)$ is the transmission range of a BS (vehicle) ignoring shadowing effect, i.e. $\sigma=0$.

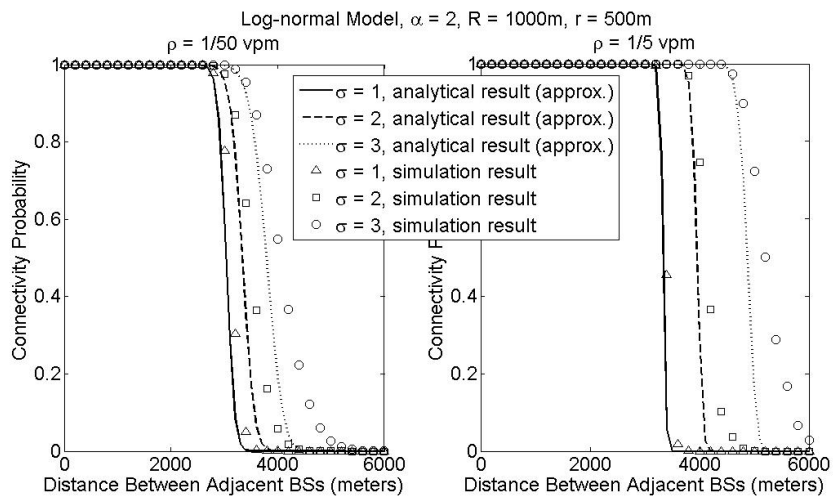

Fig. 10. Connectivity probability with $L$ changing under the log-normal model, $R=1000 \mathrm{~m}, r=500 \mathrm{~m}, \rho=1 / 5,1 / 50$ vehicles $/ \mathrm{m}$ respectively under different values of $\sigma . R(r)$ is the transmission range of a BS (vehicle) ignoring shadowing effect, i.e. $\sigma=0$.

a general connection model, and later on taking the unit disk communication model and the log-normal shadowing model as the specific examples, we derived closed-form formulas for the access probability and connectivity probability considering that the base stations and the vehicles have different transmission capabilities. These formulas characterize the relation between these key parameters, i.e. the transmission ranges of the base stations and the vehicles, the distance between adjacent base stations, the vehicle density and their impact on the access and connectivity probabilities. These results can be useful for a network operator to design a network with a given level of access guarantee. In future, we plan to extend the current work on 1-D networks to 2-D networks.

\section{REFERENCES}

[1] S. Sai, E. Niwa, K. Mase, M. Nishibori, J. Inoue, M. Obuchi, T. Harada, H. Ito, K. Mizutani, and M. Kizu, "Field evaluation of UHF radio propagation for an ITS safety system in an urban environment," IEEE Commun. Mag., vol. 47, no. 11, pp. 120-127, 2009.

[2] C.-X. Wang, X. Hong, X. Ge, X. Cheng, G. Zhang, and J. S. Thompson, "Cooperative mimo channel models: A survey," IEEE Commun. Mag., vol. 48 , no. 2 , pp. $80-87,2010$.

[3] C.-X. Wang, X. Cheng, and D. I. Laurenson, "Vehicle-to-vehicle channel modeling and measurements: recent advances and future challenges," IEEE Commun. Mag., vol. 47, no. 11, pp. 96-103, 2009.

[4] "IEEE P802.11 - task group p," http://grouper.ieee.org/groups/802/11/ Reports/tgp_update.htm. 
[5] "IEEE 1609 - family of standards for wireless access in vehicular environments (WAVE)," http://www.standards.its.dot.gov/fact_sheet.asp? $\mathrm{f}=80,2006$

[6] G. Acosta-Marum and M. A. Ingram, "Six time- and frequency- selective empirical channel models for vehicular wireless LANs," IEEE Veh. Technol. Mag., vol. 2, no. 4, pp. 4 - 11, 2007.

[7] Y. Chang, M. Lee, and J. A. Copeland, "An adaptive on-demand channel estimation for vehicular ad hoc networks," in Consumer Communications and Networking Conference (CCNC), 2009, pp. 1-5.

[8] L. Cheng, B. E. Henty, R. Cooper, D. D. Stancil, and D. F. Bai, "A measurement study of time-scaled 802.11a waveforms over the mobileto-mobile vehicular channel at $5.9 \mathrm{GHz}$," IEEE Commun. Mag., vol. 46, no. 5, pp. 84-91, 2008.

[9] L. Stibor, Y. Zang, and H.-J. Reumerman, "Evaluation of communication distance of broadcast messages in a vehicular ad-hoc network using IEEE 802.11p," in IEEE Wireless Communications and Networking Conference (WCNC), 2007, pp. $254-257$.

[10] M. Wellens, B. Westphal, and P. Mahonen, "Performance evaluation of IEEE 802.11-based WLANs in vehicular scenarios," in IEEE Vehicular Technology Conference (VTC), 2007, pp. 1167-1171.

[11] S. Eichler, "Performance evaluation of the IEEE 802.11p WAVE communication standard," in IEEE Vehicular Technology Conference (VTC), 2007, pp. 2199-2203.

[12] J. C. Burguillo-Rial, E. Costa-Montenegro, F. Gil-Castineira, and P. Rodriguez-Hernandez, "Performance analysis of IEEE 802.11p in urban environments using a multi-agent model," in IEEE International Symposium on Personal, Indoor and Mobile Radio Communications (PIMRC), 2008, pp. 1-6

[13] A. Vinel, V. Vishnevsky, and Y. Koucheryavy, "A simple analytical model for the periodic broadcasting in vehicular ad-hoc networks," in IEEE Global Communications Conference (GLOBECOM) Workshops, 2008, pp. $1-5$.

[14] A. Sebastian, M. Tang, Y. Feng, and M. Looi, "Multi-vehicles interaction graph model for cooperative collision warning system," in IEEE Intelligent Vehicles Symposium, 2009, pp. 929-934.

[15] Y. Wang, A. Ahmed, B. Krishnamachari, and K. Psounis, "IEEE $802.11 \mathrm{p}$ performance evaluation and protocol enhancement," in IEEE International Conference on Vehicular Electronics and Safety (ICVES), 2008, pp. 317-322.

[16] K. Bilstrup, E. Uhlemann, E. G. Strom, and U. Bilstrup, "Evaluation of the IEEE 802.11p MAC method for vehicle-to-vehicle communication," in IEEE Vehicular Technology Conference (VTC), 2008, pp. 1-5.

[17] J. R. Gallardo, D. Makrakis, and H. T. Mouftah, "Performance analysis of the EDCA medium access mechanism over the control channel of an IEEE $802.11 \mathrm{p}$ WAVE vehicular network," in IEEE International Conference on Communications (ICC), 2009, pp. 1-6.

[18] N. Ferreira, J. A. Fonseca, and J. S. Gomes, "On the adequacy of 802.11p MAC protocols to support safety services in ITS," in IEEE International Conference on Emerging Technologies and Factory Automation (ETFA), 2008, pp. 1189-1192.

[19] M. Amadeo, C. Campolo, A. Molinaro, and G. Ruggeri, "A WAVEcompliant MAC protocol to support vehicle-to-infrastructure non-safety applications," in IEEE International Conference on Communications (ICC) Workshops, 2009, pp. 1-6.

[20] S. Y. Wang and C. L. Chou, "NCTUns 5.0: A network simulator for IEEE $802.11(\mathrm{p})$ and 1609 wireless vehicular network researches," in IEEE Vehicular Technology Conference (VTC), 2008, pp. 1-2.

[21] T. M. Fernandez-Carames, J. A. Garcia-Naya, M. Gonzalez-Lopez, and L. Castedo, "FlexVehd: A flexible testbed for vehicular radio interfaces," in International Conference on ITS Telecommunications (ITST), 2008, pp. $283-287$.

[22] J. Peng and L. Cheng, "A distributed MAC scheme for emergency message dissemination in vehicular ad hoc networks," IEEE Trans. Veh. Technol., vol. 56, no. 6, pp. 3300 - 3308, 2007.

[23] I. Salhi, M. O. Cherif, and S. M. Senouci, "A new architecture for data collection in vehicular networks," in IEEE International Conference on Communications (ICC), 2009, pp. 1-6.

[24] M. Jonsson and A. Bohm, "Position-based data traffic prioritization in safety-critical, real-time vehicle-to-infrastructure communication," in IEEE International Conference on Communications (ICC) Workshops, 2009, pp. $1-6$.

[25] M. Torrent-Moreno, J. Mittag, P. Santi, and H. Hartenstein, "Vehicle-tovehicle communication: Fair transmit power control for safety-critical information," IEEE Trans. Veh. Technol., vol. 58, no. 7, pp. 3684 - 3703 , 2009

[26] B. Shrestha, D. Niyato, H. Zhu, and E. Hossain, "Wireless access in vehicular environments using BitTorrent and bargaining," in IEEE Global Communications Conference (GLOBECOM), 2008, pp. 1-5.
[27] J. Wu, "Connectivity analysis of a mobile vehicular ad hoc network with dynamic node population," in IEEE Global Communications Conference (GLOBECOM), 2008, pp. 1-8.

[28] O. Dousse, P. Thiran, and M. Hasler, "Connectivity in ad-hoc and hybrid networks," in IEEE Conference on Computer Communications (INFOCOM), vol. 2, 2002, pp. 1079-1088.

[29] R. S. Ojha, G. Kannan, S. N. Merchant, and U. B. Desai, "On optimal transmission range for multihop cellular networks," in IEEE Global Communications Conference (GLOBECOM), 2008, pp. 1-5.

[30] S. Mukherjee, D. Avidor, and K. Hartman, "Connectivity, power, and energy in a multihop cellular-packet system," IEEE Trans. Veh. Technol., vol. 56, no. 2, pp. 818-836, 2007.

[31] X. Ta, G. Mao, and B. D. O. Anderson, "On the connectivity of wireless multi-hop networks with arbitrary wireless channel models," IEEE Commun. Lett., vol. 13, no. 3, pp. 181 - 183, 2009.

[32] P. Agrawal and N. Patwari, "Correlated link shadow fading in multi-hop wireless networks," IEEE Trans. Wireless Commun., vol. 8, no. 8, pp. 4024-4036, 2009.

[33] C. X. Wang, M. Patzold, and Q. Yao, "Stochastic modeling and simulation of frequency-correlated wideband fading channels," IEEE Trans. Veh. Technol., vol. 56, no. 3, pp. 1050-1063, 2007.

[34] X. Cheng, C.-X. Wang, D. I. Laurenson, S. Salous, and A. V. Vasilakos, "An adaptive geometry-based stochastic model for non-isotropic mimo mobile-to-mobile channels," IEEE Trans. Wireless Commun., vol. 8, no. 9, pp. 4824-4835, 2009.

[35] C. Bettstetter and C. Hartmann, "Connectivity of wireless multihop networks in a shadow fading environment," Wireless Networks, vol. 11, no. 5, pp. 571-579, 2005.

[36] D. Miorandi, E. Alman, and G. Alfano, "The impact of channel randomness on coverage and connectivity of ad hoc and sensor networks," IEEE Trans. Wireless Commun., vol. 7, no. 3, pp. 1062-1072, 2008.

[37] X. Ta, G. Mao, and B. D. Anderson, "On the giant component of wireless multi-hop networks in the presence of shadowing," IEEE Trans. Veh. Technol., vol. 58, no. 9, pp. 5152-1563, 2009.

[38] M. Franceschetti and R. Meester, Random Networks for Communication: From Statistical Physics to Information Systems. Cambridge University Press, 2007.

Seh Chun Ng received the BEng degree in IT \& Telecommunication from University of Adelaide, Australia, and the MSc degree in IT from Malaysia University of Science and Technology (MUST), Malaysia. He is currently working toward the PhD degree in Engineering at University of Sydney. His research interest include wireless multi-hop networks and graph theory.

Wuxiong Zhang received the BEng degree in Information Security from Shanghai Jiao Tong University (SJTU), Shanghai, P.R. China, in 2008. He is now a $\mathrm{PhD}$ candidate of communication and information system in Shanghai Research Center for Wireless Communications, SIMIT, Chinese Academic of Sciences. His research interests lie in the areas of communication theory, information theory and networking. Current research focuses on ad-hoc, and vehicular networks.

Yu Zhang received her Ph.D. degree in computing from Imperial College London, UK, in 2009. She is currently working at Shanghai Engineering Research Center for Broadband Technologies and Applications, China. Her research interests include queueing theory and performance evaluation.

Yang Yang received his $\mathrm{PhD}$ degree in Information Engineering from The Chinese University of Hong Kong in 2002. He is currently working a Shanghai Research Center for Wireless Communications (WiCO), SIMIT, Chinese Academy of Sciences. Prior to that, he served the Department of Electronic and Electrical Engineering at University College London (UCL), United Kingdom, as a Senior Lecturer. His general research interests include wireless ad hoc and sensor networks, wireless mesh networks, B3G mobile communication systems.

Guoqiang Mao received $\mathrm{PhD}$ in telecommunications engineering in 2002 from Edith Cowan University. He joined the School of Electrical and Information Engineering, the University of Sydney in December 2002 where he is a Senior Lecturer now. His research interests include wireless localization techniques, wireless multihop networks, graph theory and its application in networking, and network performance analysis. He is a Senior Member of IEEE. 